\title{
The anisotropy of acoustooptic figure of merit for the collinear diffraction in $\mathrm{LiNbO}_{3}$ crystals
}

\author{
Kryvyy T., Mys O., Krupych O. and Vlokh R. \\ Vlokh Institute of Physical Optics, 23 Dragomanov Street, 79005 Lviv, Ukraine, \\ vlokh@ifo.lviv.ua
}

Received: 13.10 .2016

\begin{abstract}
We have shown that the maximal acoustooptic figure of merit for the collinear type of acoustooptic diffraction in $\mathrm{LiNbO}_{3}$ crystals is peculiar for the interaction with acoustic wave $\mathrm{QT}_{1}$ polarized parallel to the principal $Z$ axis. All the interacting waves in this case propagate in the principal plane $X Y$ along the direction inclined by 50 or $130 \mathrm{deg}$ with respect to the $X$ axis. The acoustooptic figure of merit for this interaction type is equal to $10.07 \times 10^{-15} \mathrm{~s}^{3} / \mathrm{kg}$.
\end{abstract}

Keywords: collinear acoustooptic diffraction, lithium niobate crystals, acoustooptic figure of merit, anisotropy

PACS: $43.35 . \mathrm{Sx}, 42.70 . \mathrm{Mp}$

UDC: $535.012 .2+535.42+534.321 .9$

\section{Introduction}

Collinear acoustooptic (AO) diffraction represents a particular case of $\mathrm{AO}$ interactions when the wave vectors of all the interacting waves have the same or the opposite directions. In other words, the incident and diffracted optical waves, as well as the acoustic wave (AW) are characterized by collinear wave vectors [1]. This kind of diffraction can be implemented in the two interaction geometries: (i) a transmission interaction type when all the wave vectors are parallel (the diffraction angle amounts to $\gamma=0$ ) and (ii) a reflection type when the wave vectors of the incident and diffracted optical waves are anti-parallel (the diffraction angle $\gamma=180 \mathrm{deg}$ ). The appropriate geometries are illustrated in Fig. 1. The transmission type of the collinear AO interactions can be only anisotropic, while the reflection type can be both anisotropic and isotropic. Note that the collinear AO diffraction of the reflection type is feasible at high enough $\mathrm{AW}$ frequencies only, whereas the transmission type can also be observed at low frequencies. The collinear AO diffraction is now successfully used for tunable AO filtering [2-5]. One can also remind of polarization converters constructed basing on the anisotropic collinear AO diffraction [6].

One of the principal characteristics of $\mathrm{AO}$ devices is their diffraction efficiency given by the relation [2]:

$$
\eta=\left.\sin ^{2}\left(\frac{\pi}{\lambda \cos \Theta_{B}} \sqrt{\frac{M_{2} L}{2 H} P_{a c}}\right)\right|_{\arg <<1} \simeq \frac{\pi^{2}}{\lambda^{2} \cos ^{2} \Theta_{B}} \frac{M_{2} L}{2 H} P_{a c} .
$$

Here $\lambda$ denotes the wavelength of optical radiation, $P_{a c}$ the AW power, $\Theta_{B}$ the Bragg angle, $M_{2}$ the AO figure of merit (AOFM), and $L$ and $H$ respectively the height and the width of the acoustic beam. One can see from Eq. (1) that the AO diffraction efficiency is mainly defined 
by the AOFM. The latter parameter, which has been introduced for the first time by T. M. Smith and A. Korpel [7], is defined as

$$
M_{2}=\frac{n_{k}^{3} n_{l}^{3} p_{e f}^{2}}{\rho v_{i j}^{3}} .
$$

In Eq. (2), $n_{k}$ and $n_{l}$ are the refractive indices respectively for the incident and diffractive optical waves, $p_{e f}$ denotes the effective elastooptic coefficient (EEC), $\rho$ the material density, $v_{i j}$ the velocity of the AW taking part in AO interactions, and the indices $i$ and $j$ correspond respectively to the directions of propagation and polarization of the AW. In case of the collinear interactions, Eq. (1) reads as

$$
\eta=\sin ^{2}\left(l \sqrt{\frac{M_{2} \pi^{2}}{2 \lambda^{2} A} P_{a c}}\right),
$$

where $A=L H$ is the acoustic-beam area and $l$ is the interaction length [5].

Usually the collinear AO diffraction is associated with the interacting waves that propagate along the principal crystallographic axes and, therefore, it deals with the EECs determined by the elastooptic tensor components like $p_{41}, p_{45}, p_{51}, p_{52}, p_{54}, p_{56}, p_{62}, p_{64}$ and $p_{65}[1,8]$. The matrix of elastooptic coefficients for the $\mathrm{LiNbO}_{3}$ crystals belonging to the point symmetry group $3 \mathrm{~m}$ also contains the relevant component, $p_{41}$. However, it is very small for $\mathrm{LiNbO}_{3}$ $(-0.051 \pm 0.011$ [9]) and, as follows from the results presented in this paper, the appropriate AOFM is as low as $1.15 \times 10^{-15} \mathrm{~s}^{3} / \mathrm{kg}$. We are to stress in this respect that the collinear AO diffraction can become efficient enough only in the case when the EEC is not too small (e.g., $\geq 0.05[1])$.

Moreover, if we restrict ourselves to the propagation of waves along the crystallographic axes, the geometry of anisotropic collinear interaction that relies on the coefficient $p_{e f}=p_{41}$ is strictly limited: all of the waves should propagate along the $b$ axis. On the other hand, it is known from the literature (see Ref. [5]) that the collinear geometry can yield in the AOFM as large as $7.5 \times 10^{-15} \mathrm{~s}^{3} / \mathrm{kg}$ in the case of propagation of the interacting waves close to the optic axis (20 deg with respect to the $c$ axis in the $b c$ plane). Irrespective of its reliability (see below), this result certainly hints that, among different collinear AO interaction geometries, one can expect to find the geometries more efficient than a standard one that corresponds to propagation of the waves along the crystallographic axes. This resource stems from anisotropy of AO properties, which must be peculiar for any crystalline material.

In the present work we will analyze the AOFM anisotropy for the collinear AO interactions occurring in a canonical nonlinear optical and $\mathrm{AO}$ crystal, lithium niobate. $\mathrm{LiNbO}_{3}$ is now efficiently used for constructing collinear mode converters [10-12], Bragg cells [13] and AO tunable filters [14]. In the most of cases, these devices are used as AO waveguides based on integrated-optical principles, since lithium niobate represents a well-known material for fabricating planar or channel waveguides. Notice that the interaction geometry in these devices has earlier been chosen from the viewpoints of technology [13], bandwidth versus efficiency and optical wavelength versus efficiency optimizations $[11,12]$. Concerning a further optimization of the collinear AO interaction geometry from the standpoint of AOFM anisotropy, the authors know of 
the only work [5], where the anisotropy of $\mathrm{AO}$ properties in $\mathrm{LiNbO}_{3}$ has been considered in the $b c$ plane. The authors of Ref. [5] have found that the maximal AOFM $\left(\sim 17 \times 10^{-15} \mathrm{~s}^{3} / \mathrm{kg}\right)$ can be reached when the waves propagate approximately at the angle -25 deg with respect to the $b$ axis in the $b c$ plane. Unfortunately, the other interaction geometries have not been considered. As a result, the scope of the present work includes the analysis of anisotropy of the AOFM for the case of collinear AO diffraction, using lithium niobate as both important and practical example.
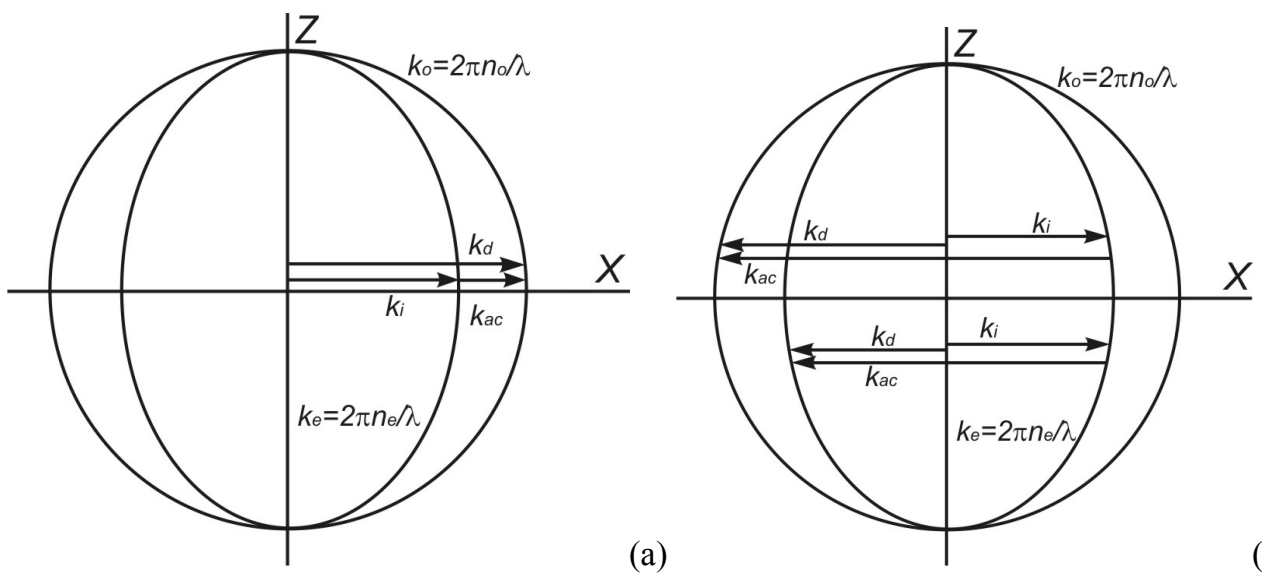

(b)

Fig. 1. Two geometries of collinear $A O$ interactions denoted as (a) transmission and (b) reflection types. $k_{i}$, $k_{d}$ and $K_{a c}$ are the wave vectors of incident, diffracted and acoustic waves, respectively.

\section{Results and discussion}

Let us analyze the AOFM anisotropy using a theoretical method developed in Refs. [15, 16] for the trigonal crystals that belong to the symmetry groups $3 \mathrm{~m}, 32$ and $\overline{3} \mathrm{~m}$. One of the mirror symmetry planes in $\mathrm{LiNbO}_{3}$ is perpendicular to the crystallographic axis $a$ [17], while the $c$ axis is parallel to the three-fold symmetry axis. Here the crystallographic axes $a, b$ and $c$ correspond respectively to the $X, Y$ and $Z$ axes of Fresnel ellipsoid. The refractive indices at the optical wavelength $\lambda=632.8 \mathrm{~nm}$ are equal to $n_{o}=2.286$ and $n_{e}=2.203$ [17], i.e. $\mathrm{LiNbO}_{3}$ is optically negative $\left(n_{o}>n_{e}\right)$. The elastooptic coefficients at the same wavelength are as follows [9]: $p_{11}=-0.023 \pm 0.017, \quad p_{12}=0.076 \pm 0.014, \quad p_{13}=0.147 \pm 0.019, \quad p_{31}=0.157 \pm 0.007$, $p_{33}=0.141 \pm 0.013, p_{14}=0.057 \pm 0.004, p_{41}=-0.051 \pm 0.011$ and $p_{44}=0.126 \pm 0.004$. Finally, the elastic stiffness coefficients under the conditions of constant electric field are equal to $C_{11}=2.03$, $C_{12}=0.573, C_{13}=0.752, C_{33}=2.42, C_{44}=0.595, C_{66}=0.728$ and $C_{14}=0.085$ (all in the units of $10^{11} \mathrm{~N} / \mathrm{m}^{2}$ ), and the density of $\mathrm{LiNbO}_{3}$ amounts to $\rho=4640 \mathrm{~kg} / \mathrm{m}^{3}[18]$.

The relations for the EEC suitable for the anisotropic diffractions have been derived elsewhere $[15,16]$. They correspond to the interactions with quasi-longitudinal (QL) and two quasi-transverse $\left(\mathrm{QT}_{1}\right.$ and $\left.\mathrm{QT}_{2}\right)$ waves. These interactions correspond to so-called types VII, VIII and IX, respectively (see in Refs. $[15,16]$ ). The wave $\mathrm{QT}_{1}$ is polarized in the interaction plane $X Z$ parallel to the $Z$ axis, and the polarization of the wave $\mathrm{QT}_{2}$ is perpendicular to this plane.

In this work we concentrate on the transmission type of anisotropic collinear diffraction, for which the diffraction angle is equal to $\gamma=0$. We obtain the following relations for the interaction types VII, VIII and IX: 
త

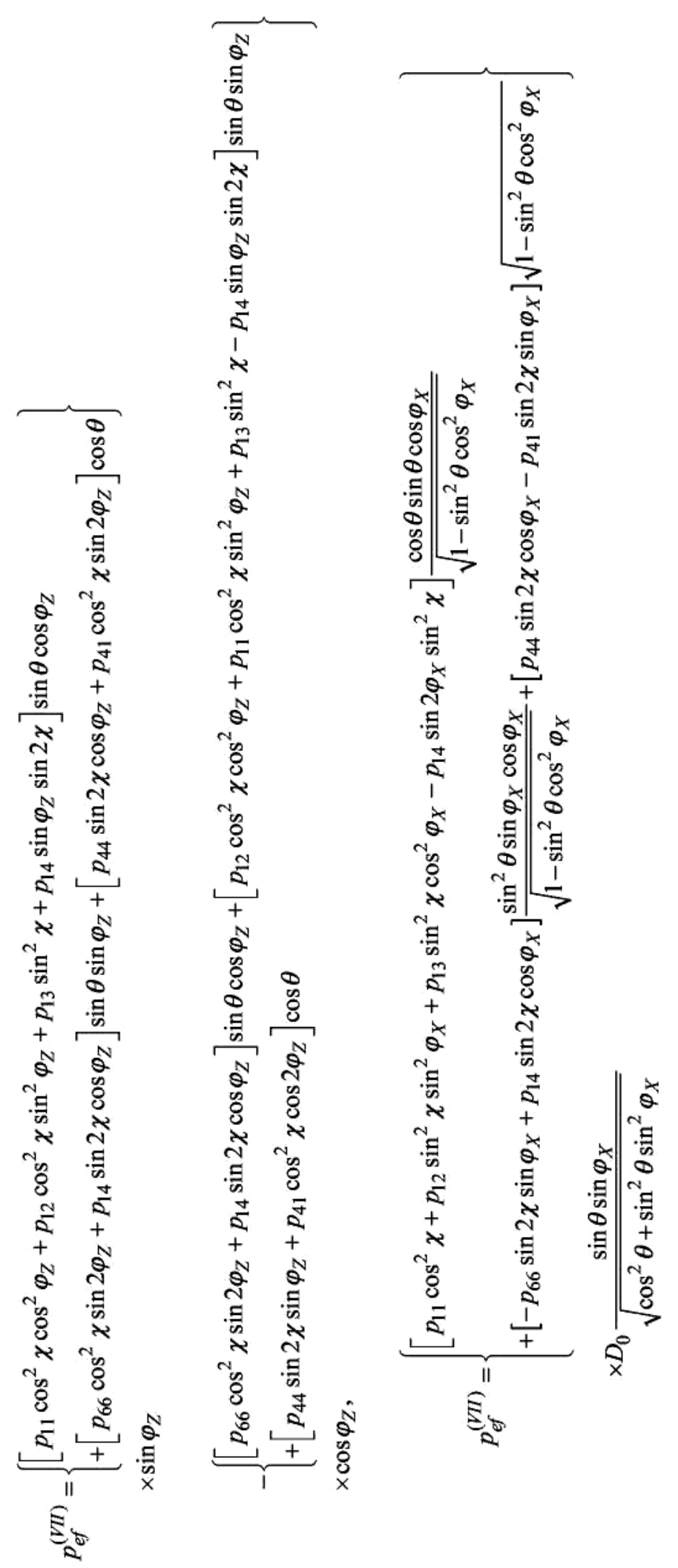

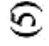

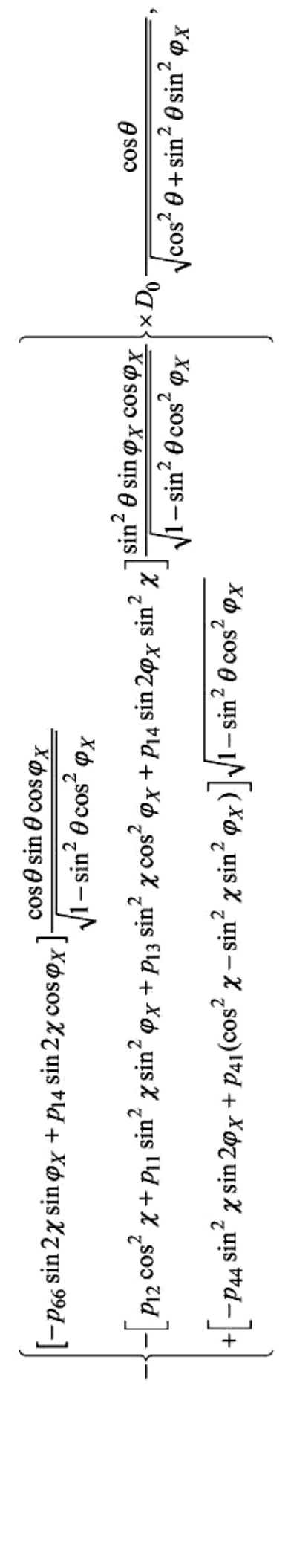

Ukr. J. Phys. Opt. 2016, Volume 17, Issue 4 
e

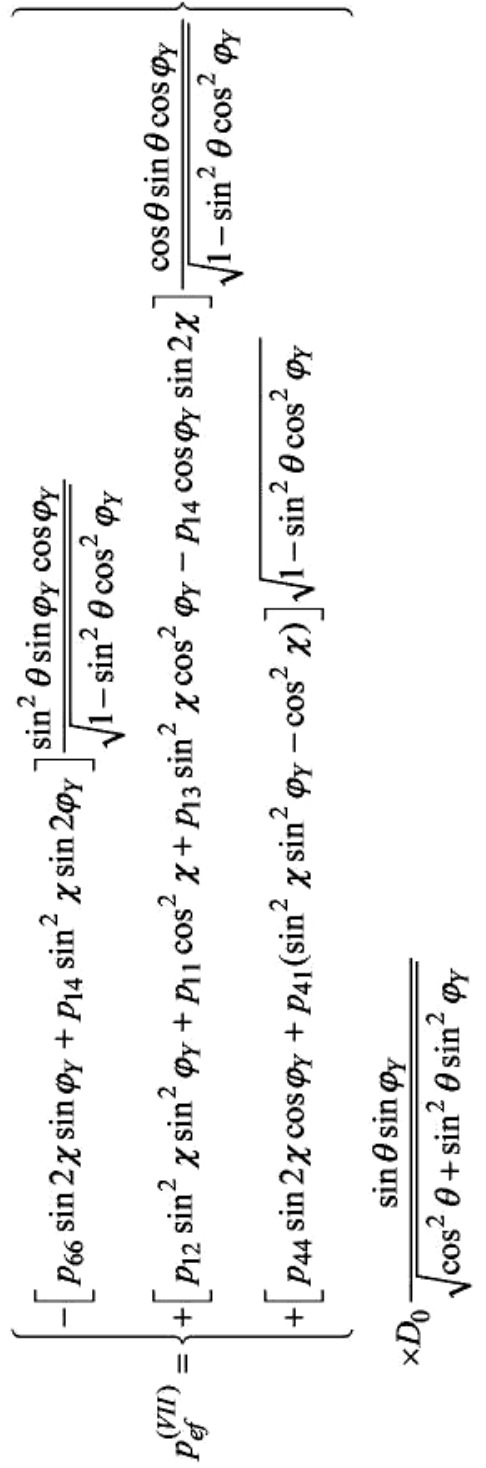

$\varepsilon$

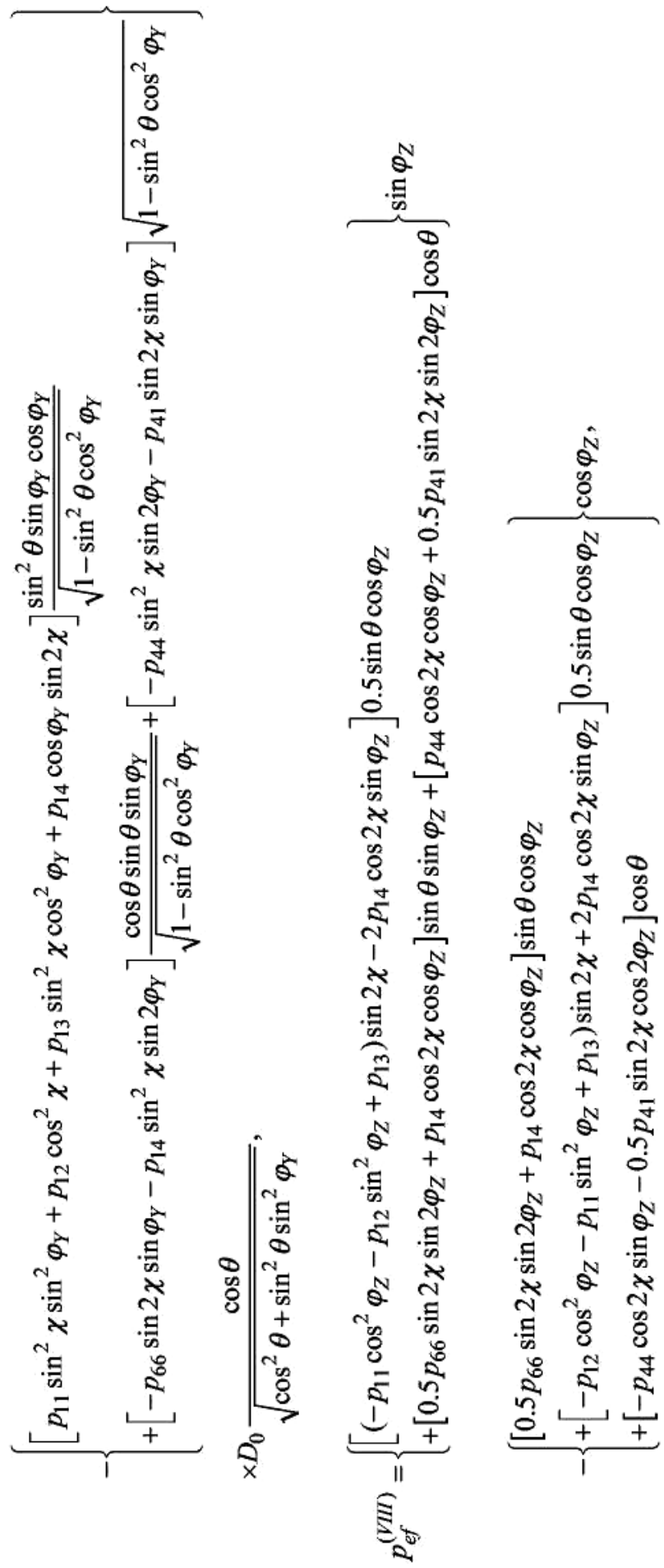


ळ

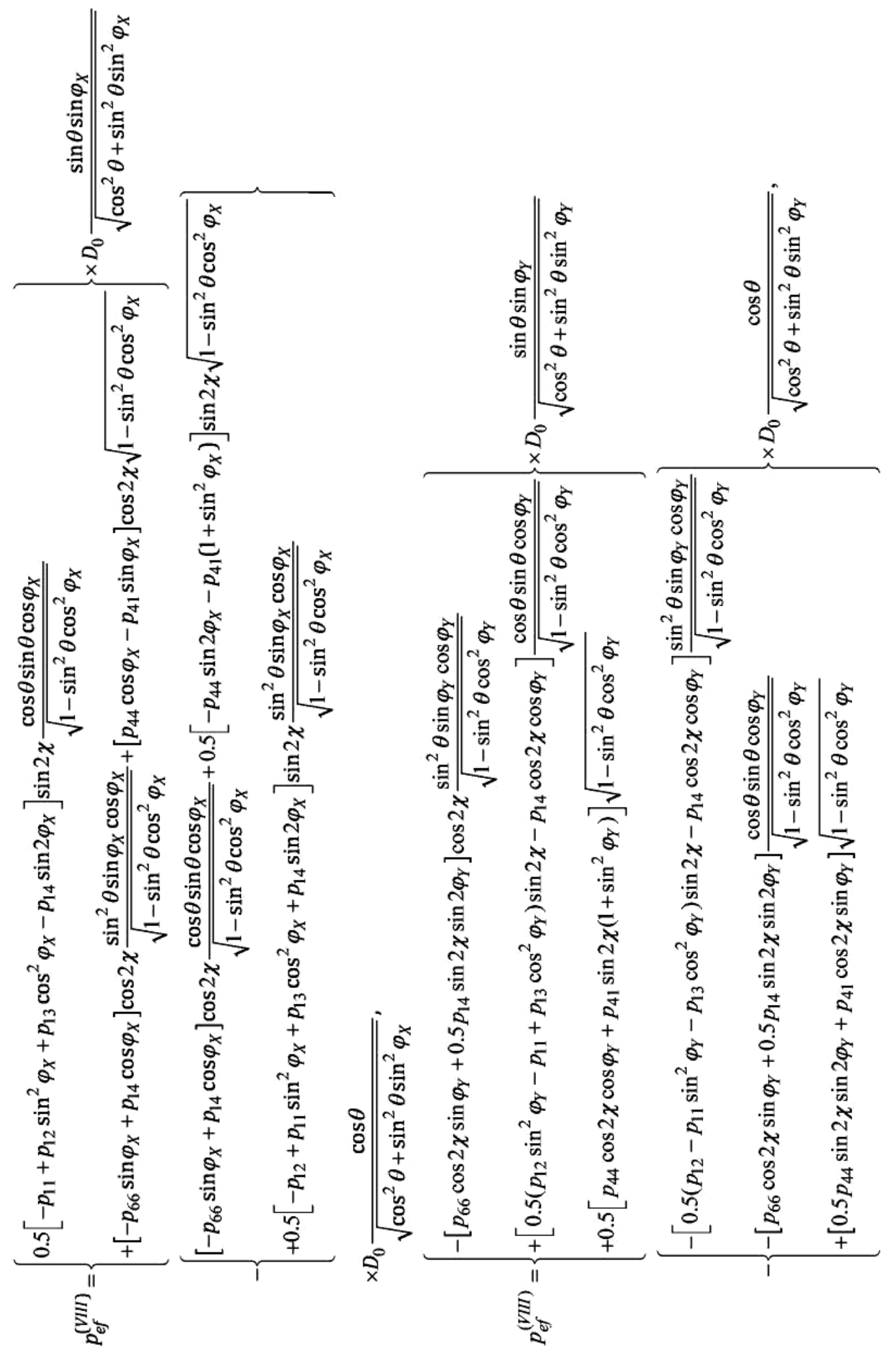

Ukr. J. Phys. Opt. 2016, Volume 17, Issue 4 


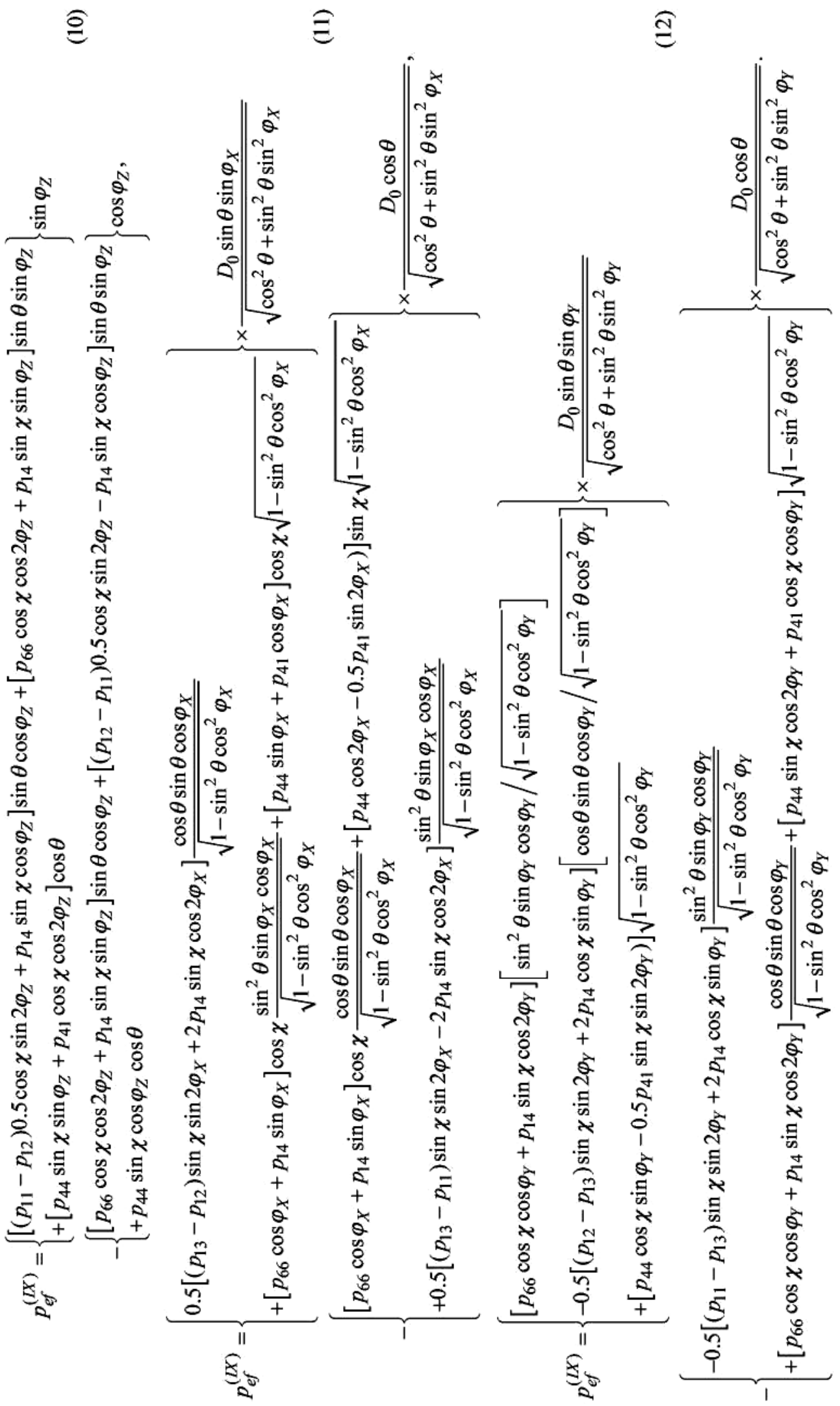


In Eqs. (4)-(12), the angles $\varphi_{X}$ and $\varphi_{Z}$ determine the rotations of the interaction plane $X Z$ respectively around the $X$ and $Z$ axes, the angle $\varphi_{Y}$ describes the rotation of the interaction plane $Y Z$ around the $Y$ axis, and $\theta$ is the incident angle of the optical wave with respect to the $X$ (or $Y$ ) axis. The orientation angle of the AW vector with respect to the $X$ (or $Y$ ) axis for optically negative crystals is given by

$$
\chi=\arctan \left(\frac{n_{o} \sin (\theta+\gamma)-n_{o}^{*} n_{e} \frac{\sin \theta}{\sqrt{n_{o}^{* 2} \cos ^{2} \theta+n_{e}^{2} \sin ^{2} \theta}}}{n_{o} \cos (\theta+\gamma)-n_{o}^{*} n_{e} \frac{\cos \theta}{\sqrt{n_{o}^{* 2} \cos ^{2} \theta+n_{e}^{2} \sin ^{2} \theta}}}\right) .
$$

The parameter $n_{o}^{*}$ in Eq. (13) depends on the angles of rotation of the interaction plane $\varphi_{X}$ and $\varphi_{Y}$ around the $X$ and $Y$ axes:

$$
n_{o}^{*}=\frac{n_{o} n_{e}}{\sqrt{n_{e}^{2} \cos ^{2}\left(\varphi_{X} \text { or } \varphi_{Y}\right)+n_{o}^{2} \sin ^{2}\left(\varphi_{X} \text { or } \varphi_{Y}\right)}} .
$$

Then the relations for the AOFM read as

$$
M_{2}^{(V I I)}=\frac{n_{o}^{3} n_{e}^{* 3}\left\{p_{e f}^{(V I I)}\right\}^{2}}{\rho\left[v_{Q L}\left(\chi, \varphi_{X, Y, Z}\right)\right]^{3}}, M_{2}^{(V I I I)}=\frac{n_{o}^{3} n_{e}^{* 3}\left\{p_{e f}^{(V I I I)}\right\}^{2}}{\rho\left[v_{Q T_{1}}\left(\chi, \varphi_{X, Y, Z}\right)\right]^{3}}, M_{2}^{(I X)}=\frac{n_{o}^{3} n_{e}^{* 3}\left\{p_{e f}^{(I X)}\right\}^{2}}{\rho\left[v_{Q T_{2}}\left(\chi, \varphi_{X, Y, Z}\right)\right]^{3}},(
$$

where $v_{Q L}\left(\chi, \varphi_{X, Y, Z}\right), v_{Q T_{1}}\left(\chi, \varphi_{X, Y, Z}\right)$ and $v_{Q T_{2}}\left(\chi, \varphi_{X, Y, Z}\right)$ define the changes in the AW velocity occurring in different interaction planes. Finally, the modified refractive indices become as follows:

$$
n_{e}^{*}=\frac{n_{o} n_{e}}{\sqrt{n_{o}^{2} \cos ^{2} \theta+n_{e}^{2} \sin ^{2} \theta}}, \quad n_{e}^{*}=\frac{n_{o}^{*} n_{e}}{\sqrt{\left(n_{o}^{* 2}-n_{e}^{2}\right) \cos ^{2} \varphi_{X}\left(\text { or } \varphi_{Y}\right) \sin ^{2} \theta+n_{e}^{2}}}
$$

It is worthwhile that the momentum conservation law in a general case of non-collinear AO diffractions involves a triangle of interacting wave vectors. Therefore, the AOFM anisotropy must be considered for different interaction planes defined by the triangle mentioned. On the other hand, it is enough to consider the interaction directions, instead of the interaction planes, in the case of collinear diffraction. In the latter case, one can analyze the rotations of the interaction planes determined by Eqs. (4)-(12) with respect to a single axis, e.g., the axis $Z$. Due to the three-fold axial symmetry of the $\mathrm{LiNbO}_{3}$ crystals, it is enough to change the $\varphi_{Z}$ angle by $120 \mathrm{deg}$ with respect to the $Y$ axis, or by $150 \mathrm{deg}$ with respect to the $X$ axis. Completing consideration of these rotation ranges by the analysis of the overall change 0-360 deg in the incidence angle $\theta$, one can be sure in covering the whole solid angle $4 \pi$ sr of any possible interaction directions.

Below we report in brief the main results of our analyses for the dependences of AOFM and EEC on the $\theta$ angle at different $\varphi_{Z}$ angles (see Fig. 2, Fig. 3 and Fig. 4). As seen from Fig. 2, the AOFM is very small for the type VII of AO interactions with the AW QL: its maximal value is only $0.25 \times 10^{-15} \mathrm{~s}^{3} / \mathrm{kg}$ at $\varphi_{Z}=0,60$ or $120 \mathrm{deg}$ and $\theta=0 \mathrm{deg}$. This AOFM corresponds to the propagation of the interacting waves along the $X$ axis or along the direction symmetrically identical to the $X$ axis in the $X Y$ plane.

Ukr. J. Phys. Opt. 2016, Volume 17, Issue 4 

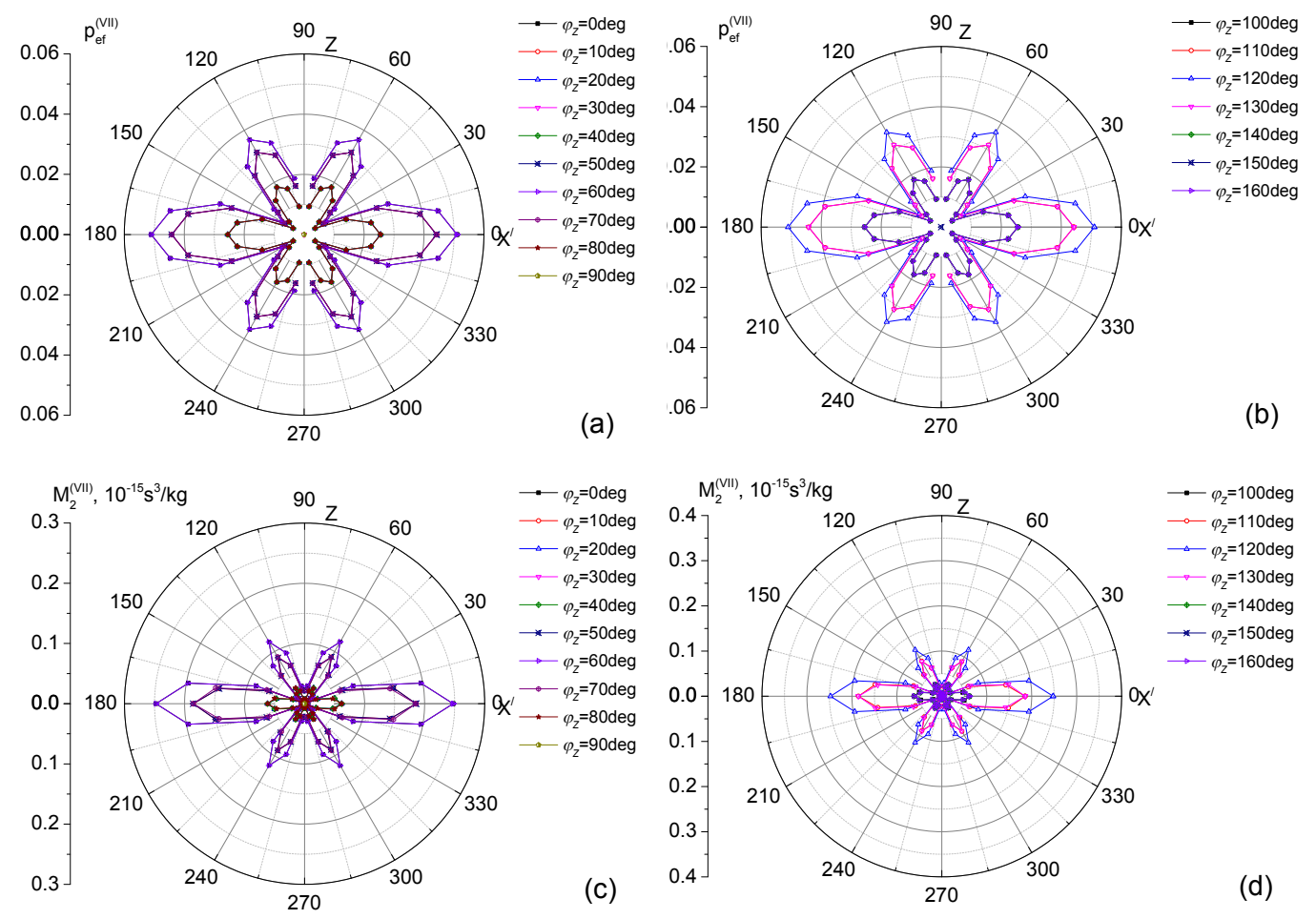

Fig. 2. Dependences of EEC and AOFM on the incident angle $\theta$ for the type VII of collinear AO interactions in $\mathrm{LiNbO}_{3}$.
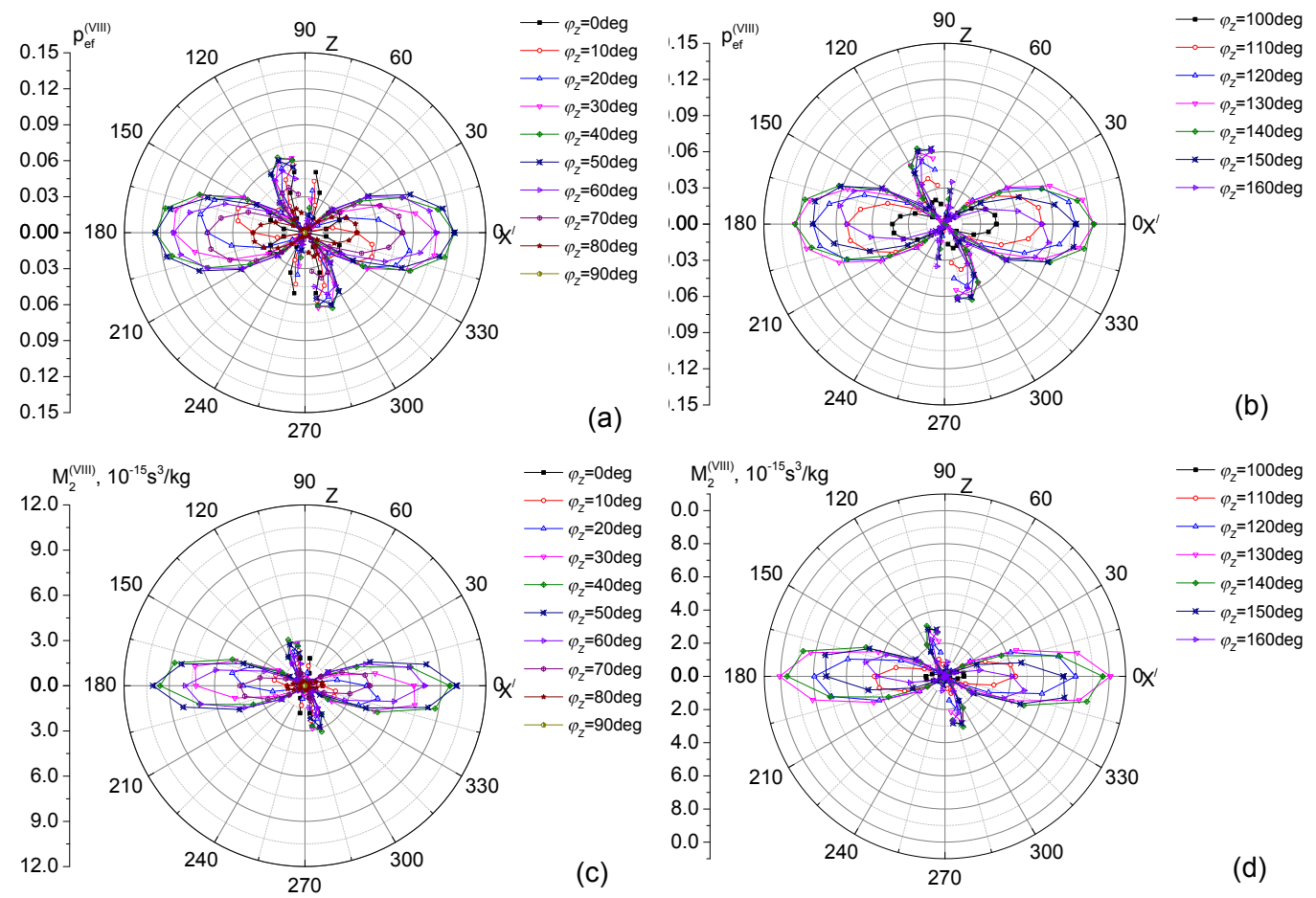

Fig. 3. Dependences of EEC and AOFM on the incident angle $\theta$ for the type VIII of collinear AO interactions in $\mathrm{LiNbO}_{3}$. 
For the type VIII of AO interactions, the maximal AOFM $\left(10.07 \times 10^{-15} \mathrm{~s}^{3} / \mathrm{kg}\right)$ is peculiar to the interactions with the $\mathrm{AW} \mathrm{QT}_{1}$ (see Fig. 3). It is achieved at $\varphi_{Z}=50 \mathrm{deg}$ (or $130 \mathrm{deg}$ ) and $\theta=0$ deg. The maximal AOFM value is reached when the optical and acoustic waves propagate in the $X Y$ plane along the direction that deviates from the crystallographic axes. Finally, the maximal AOFM for the type IX of interactions with the AW $\mathrm{QT}_{2}$ is equal to $3.5 \times 10^{-15} \mathrm{~s}^{3} / \mathrm{kg}$ (see Fig. 4). This AOFM characterizes the interaction of waves propagating in the $X Z$ plane ( $\varphi_{Z}=0 \mathrm{deg}$ ) at the $\theta$ angles equal to $45,135,225$ or $315 \mathrm{deg}$.
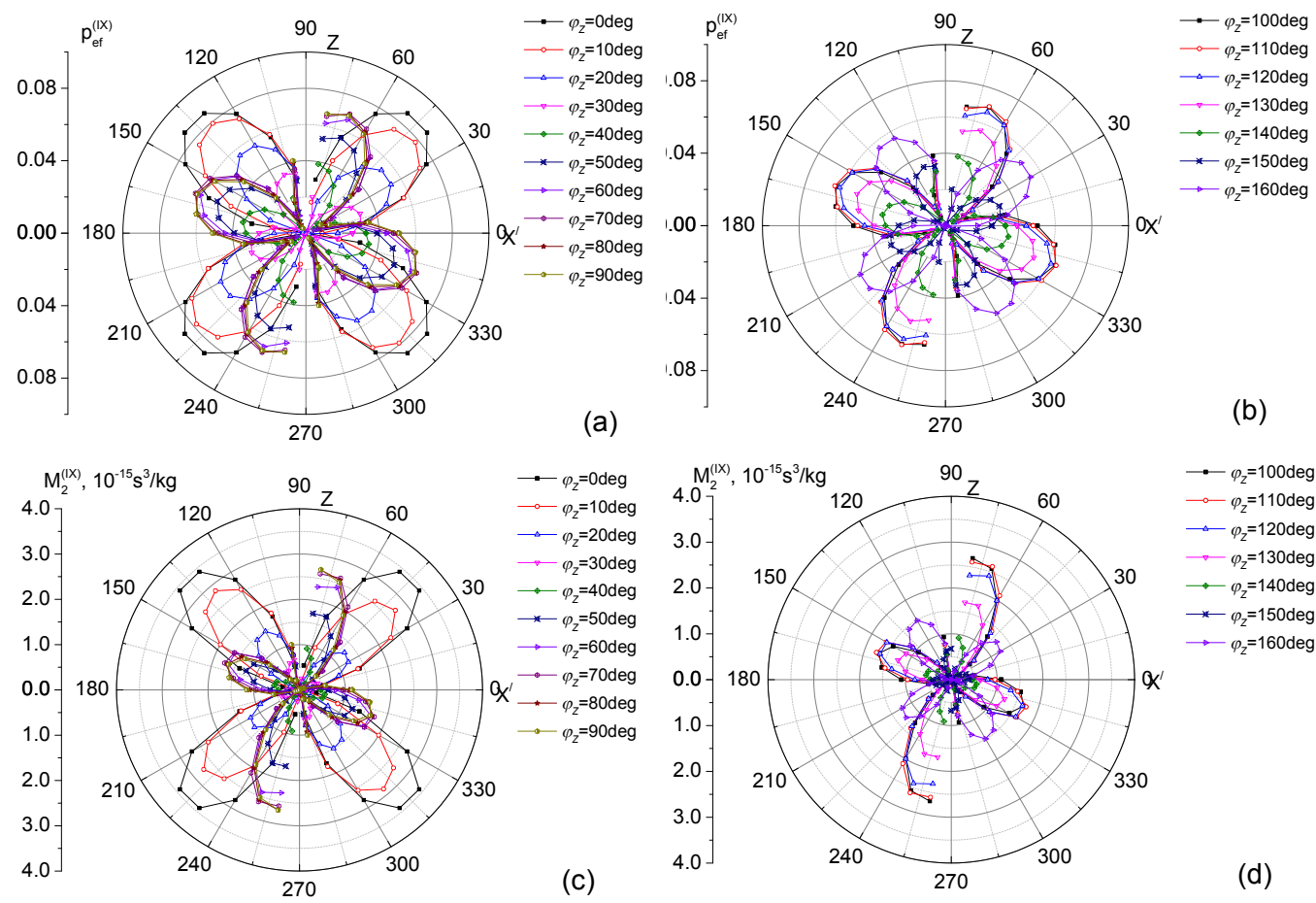

Fig. 4. Dependences of EEC and AOFM on the incident angle $\theta$ for the type IX of collinear AO interactions in $\mathrm{LiNbO}_{3}$.

Hence, the global AOFM maximum for $\mathrm{LiNbO}_{3}$ occurring in the case of collinear AO diffraction is reached if one deals with the $\mathrm{AO}$ interactions with the $\mathrm{AW} \mathrm{QT}_{1}$ (i.e., the type VIII). Of course, this AOFM value is not so high if compared with the best AO materials (see, e.g., Refs. [19, 20]). Nonetheless, for the case of collinear interaction this AOFM can lead to a $100 \%$ efficiency at the interaction lengths $10^{-1} \mathrm{~m}, A=25 \mathrm{~mm}^{2}, \lambda=632.8 \mathrm{~nm}$ and the AW powers as small as $5 \times 10^{-2} \mathrm{~W}$.

As follows from Fig. 2, Fig. 3 and Fig. 4, the anisotropy of AOFM is determined by the anisotropy of EEC. Notice also that our results differ from those presented in the work [5]. For example, we predict the AOFM $2.5 \times 10^{-15} \mathrm{~s}^{3} / \mathrm{kg}$ (the interaction type XI, $\varphi_{Z}=90 \mathrm{deg}$ and $\theta=70 \mathrm{deg}$ ) for the geometry of collinear interactions at which the interacting waves propagate close to the optic axis (20 deg with respect to the $c$ axis in the $b c$ plane), whereas the authors [5] report somewhat larger value $\left(7.5 \times 10^{-15} \mathrm{~s}^{3} / \mathrm{kg}\right)$. Moreover, according to our data, the AOFM value equal to $\sim 17 \times 10^{-15} \mathrm{~s}^{3} / \mathrm{kg}$ cannot be reached with $\mathrm{LiNbO}_{3}$ in the case of collinear $\mathrm{AO}$ interactions. The differences among our results and those of Ref. [5] are, most probably, caused by different elastooptic tensor components used in AOFM calculations. For instance, the EEC 
characterizing $\mathrm{AO}$ interactions in the plane $b c$ (the propagation direction is inclined by 20 deg with respect to the $c$ axis) is equal to -0.108 [5], although, according to our data, it is notably less $\left(\left|p_{e f}\right|=0.07\right)$. Unfortunately, we cannot directly compare our results for the maximal AOFMs with the experimental data since, for the best of our knowledge, the experimental results on the efficiency of collinear AO interactions in the $X Y$ plane of $\mathrm{LiNbO}_{3}$ have not yet been presented in the literature.

\section{Conclusions}

In the present work we have analyzed the anisotropy of AOFM for the collinear AO interactions occurring in the $\mathrm{LiNbO}_{3}$ crystals. The analysis has been performed for the three types of anisotropic interactions, those with the $\mathrm{AWs}$ termed as $\mathrm{QL}, \mathrm{QT}_{1}$ and $\mathrm{QT}_{2}$. We have found that the maximal AOFM is peculiar for the type VIII of interactions with the AW $\mathrm{QT}_{1}$, which is polarized parallel to the principal $Z$ axis. Under the conditions of this diffraction, all the interacting waves propagate in the principal plane $X Y$ along the direction that makes the angles 50 or $130 \mathrm{deg}$ with respect to the $X$ axis. The AOFM value is then equal to $10.07 \times 10^{-15} \mathrm{~s}^{3} / \mathrm{kg}$. We have also shown that the anisotropy of AOFM for the case of collinear diffraction is determined by the EEC anisotropy.

\section{References}

1. Balakshii V I, Parydin VN and Chirkov LE, Physical fundamentals of acoustooptics. Moscow: Radio i Svyaz (1985).

2. Magdich L N and Molchanov V Ya, Acoustooptic devices and their application. New York: Gordon and Breach Science Publishing (1989).

3. Morasca S, Scarano D and Schmid S, 1997. Application of $\mathrm{LiNbO}_{3}$. Acoustooptic tunable switches and filters in WDM transmission networks at high bit rates. In: Book of photonic networks, ed. by G Prati, Part 3. Advances in Optical Communications (pp. 458-472).

4. Nakazawa T, Taniguchi S and Seino M, 1999. Ti: $\mathrm{LiNbO}_{3}$ acousto-optic tunable filter (AOTF). FUJITSU Sci. Techn. J. 35: 107-112.

5. Fujii $\mathrm{Y}$ and Hayashi H, 1977. Acousto-optic tunable filter using $\mathrm{LiNbO}_{3}$ crystals. Proc. SPIE. 99: 110-115.

6. Yudistira D, Janner D, Benchabane $\mathrm{S}$ and Pruneri V, 2009. Integrated acousto-optic polarization converter in a $\mathrm{ZX}$-cut $\mathrm{LiNbO}_{3}$ waveguide superlattice. Opt. Lett. 34: 3205-3207.

7. Smith T and Korpel A, 1965. Measurement of light-sound interaction efficiencies in solids. IEEE J. Quant. Electron. 1: 283-284.

8. Kusters J A, Wilson D A and Hammond D L, 1974.Optimum crystal orientation for acoustically tuned optical filters. J. Opt. Soc. Amer. 64: 434-440.

9. Krupych O, Savaryn V and Vlokh R, 2014. Precise determination of full matrix of piezo-optic coefficients with a four-point bending technique: the example of lithium niobate crystals. Appl. Opt. 53: B1-B7.

10. Binh L N and Livingstone J, 1980. Optimisation of a collinear acoustooptic TEm-TEn mode convertor $\mathrm{LiNbO}_{3}$. IEE Proc. (H - Microwaves, Optics and Antennas). 127: 323-329.

11. Petrov D V and Čtyroký J, 1985. Optimal parameters of single-mode $\mathrm{LiNbO}_{3}$ :Ti waveguides for collinear acoustooptic Interaction. Sov. J. Quant. Electron. 15: 58-60.

12. Hinkov I and Hinkov V, 1993. Acoustooptic collinear TE-TM mode conversion in a two-layer Ti-indiffused and proton-exchanged waveguide structure in $\mathrm{LiNbO}_{3}$. Opt. Quant. Electon. 25: 195-200. 
13. Palma F and Schirone L, 1986. Acoustooptic interaction efficiency in Ti: $\mathrm{LiNbO}_{3}$ waveguide collinear Bragg diffraction cell. J. Appl. Phys. 60: 3720-3723.

14. S. E. Harris and R. W. Wallace, 1969. Acousto-optic tunable filter. J. Opt. Soc. Amer. 59: $744-747$.

15. Mys O, Kostyrko M and Vlokh R, 2016. The anisotropy of acousto-optic figure of merit for $\mathrm{LiNbO}_{3}$ crystals: Anisotropic diffraction. Appl. Opt. 55: 2439-2450.

16. Mys O, Krupych O, Kostyrko M and Vlokh R, 2016. Anisotropy of acousto-optic figure of merit for $\mathrm{LiNbO}_{3}$ crystals: anisotropic diffraction. Errata. Appl. Opt. (to be published).

17. Weis R S and Gaylord T K, 1985. Lithium niobate: summary of physical properties and crystal structure. Appl. Phys. A. 37: 191-203.

18. Smith R T and Welsh F S, 1971. Temperature dependence of the elastic, piezoelectric, and dielectric constants of lithium tantalate and lithium niobate. J. Appl. Phys. 42: 2219-2230.

19. Vlokh R and Martynyuk-Lototska I, 2009. Ferroelastic crystals as effective acoustooptic materials. Ukr. J. Phys. Opt. 10: 89-99.

20. Martynyuk-Lototska I Y, Mys O G, Grabar A A, Stoika I M, Vysochanskii Y M and Vlokh R O. Highly efficient acousto-optic diffraction in $\mathrm{Sn}_{2} \mathrm{P}_{2} \mathrm{~S}_{6}$ crystals. Appl. Opt. 2008 47: 52-55.

Kryvyy T., Mys O., Krupych O. and Vlokh R. 2016. The anisotropy of acoustooptic figure of merit for the collinear diffraction in $\mathrm{LiNbO}_{3}$ crystals. 17: $176-187$

Анотація. Показано, щзо максимальне значення коефіцієнта акустооптичної якості для колінеарної акустооптичної дифракиії в кристалах ніобату літію притаманне взаємодії з акустичною хвилею $Q T_{1}$, поляризованою паралельно до головної осі $Z$. Тодi всі взаємодіючі хвилі поширюються в головній плошині ХY у напрямках, щзо відхиляються від осі X на кути 50 і 130 град. Відповідне значення коефіиієнта акустооптичної якості складас $10,07 \times 10^{-15} \mathrm{c}^{3} / \kappa 2$. 